\title{
REMOÇÃO DE CORANTE TEXTIL UTILIZANDO A CASCA DO ABACAXI COMO ADSORVENTE NATURAL
}

\author{
E. C. E. S. ANTUNES ${ }^{1}$, J. E. S. PEREIRA ${ }^{2, ~}{ }^{*}$, R. L. S. FERREIRA ${ }^{3}$, M. F. D. MEDEIROS ${ }^{4}$, E. L. BARROS NETO ${ }^{5}$ \\ ${ }^{1,4}$ Departamento de Engenharia Química - Universidade Federal do Rio Grande do Norte \\ 2,5 Programa de Pós-Graduação em Engenharia Química - Universidade Federal do Rio Grande do Norte \\ ${ }^{3}$ Programa de Pós-Graduação em Engenharia Civil - Universidade Federal do Rio Grande do Norte \\ jessycaemanuella@hotmail.com ${ }^{*}$
}

Submetido 10/11/2016 - Aceito 30/08/2018

DOI: $10.15628 /$ holos.2018.5334

\section{RESUMO}

Este trabalho avaliou a utilização da casca de abacaxi (Ananas comosus) como adsorvente para remoção do corante catiônico azul de metileno presente em solução aquosa. $\mathrm{O}$ adsorvente foi obtido através da secagem e trituração das cascas da fruta. A granulometria do material foi $<0.2 \mathrm{~mm}$. Os ensaios de adsorção foram realizados em batelada, sob rotação de $150 \mathrm{rpm}$ e à temperatura de 30 e $40^{\circ} \mathrm{C}$. O efeito da quantidade de adsorvente $(0,1 \mathrm{~g}-0,5 \mathrm{~g})$ e concentração inicial de adsorbato (60ppm-100ppm) no percentual de remoção (\%R) e na capacidade de adsorção unitária (qe) foram avaliados. Os modelos de isoterma de Langmuir e Freundlich foram utilizados na descrição matemática do sistema. A casca de abacaxi apresentou remoção de até $81 \%$ do corante azul de metileno. 0 aumento da massa de adsorvente proporcionou um aumento na percentagem de remoção do corante e diminuição da capacidade de adsorção unitária (qe). O aumento da concentração inicial do adsorbato em solução aquosa provocou uma redução na percentagem de remoção e um aumento na capacidade de adsorção unitária (qe). 0 modelo de isoterma de Freundlich foi o que melhor se ajustou aos dados experimentais. $\mathrm{O}$ ajuste do modelo de Langmuir aos dados experimentais revelou a capacidade de adsorção unitária máxima para a casca de abacaxi como sendo de $17,12 \mathrm{mgg}^{-1}$ e $10,06 \mathrm{mgg}^{-1}$ à $30 \mathrm{C}$ e $40^{\circ} \mathrm{C}$, respectivamente. $\mathrm{O}$ caráter da reação de adsorção foi exotérmico pois a elevação da temperatura desfavoreceu a remoção do corante. Os resultados apontam que esse resíduo agroindustrial tem potencial para ser utilizado como um adsorvente econômico para a remoção de azul de metileno.

PALAVRAS-CHAVE: Resíduo agroindustrial, abacaxi, adsorção, azul de metileno.

\section{DYE REMOVAL USING PINEAPPLE PEEL AS A NATURAL ADSORBENT}

\begin{abstract}
This work aimed to study the use of pineapple peel (Ananas comosus) as adsorbent for methylene blue (MB) removal from aqueous solution. The adsorbent was obtained by drying and grinding the fruit peel. The particle size of the material was $<0.2 \mathrm{~mm}$. Batch adsorption experiments were carried out at speed rotation of $150 \mathrm{rpm}$ and under two temperature conditions (30 and $40{ }^{\circ} \mathrm{C}$ ). The effect of initial dye concentration (60 ppm - 100 ppm) and adsorbent dose $(0.1 \mathrm{~g}-0.5 \mathrm{~g})$ on the adsorption unity capacity (qe) and percentage removal of methylene blue (\%R) were investigated. The biosorbent obtained from the pineapple peel showed satisfactory results, removing up
\end{abstract}

to $81 \%$ of adsorbate from the solution. The percentage removal of methylene blue increased with the increasing of adsorbent mass and decreased as the initial dye concentration was increased. The adsorption unity capacity was higher at higher initial dye concentration conditions and lower at higher adsorbent dose. The adsorption was favorable at lower temperature and the equilibrium data were well fitted by the Langmuir model. The maximum adsorption capacity ranged from $17.12 \mathrm{mg}$ g-1 to $10.06 \mathrm{mg}$ g-1 as the temperature increased from $30 \mathrm{C}$ to $40^{\circ} \mathrm{C}$. The results showed that this agricultural residue has potential to be used as an economical adsorbent for removal of methylene blue.

KEYWORDS: Residue agroindustrial, pineapple, adsorption, methylene blue. 


\section{INTRODUÇÃO}

O crescimento populacional e, consequentemente, o aumento pela demanda de alimentos tem impulsionado o setor agroindustrial, o qual representa uma das principais parcelas da economia brasileira. Nesse sentido, estima-se que a agroindústria represente $30 \%$ da economia brasileira e envolva grande parte dos setores econômicos em que o Brasil apresenta expressiva competitividade no cenário internacional. Dentre os diversos setores que compõe o agronegócio brasileiro, destaca-se o da fruticultura devido à grande variedade de culturas produzidas em todo o país (Nascimento, 2006).

Segundo dados do Serviço Brasileiro de Apoio à Micro e Pequenas Empresas - SEBRAE (2015) e Pathak, Mandavgane e Kulkarni (2015), no cenário internacional, o Brasil ocupa a terceira posição no ranking dos países produtores de frutas, ficando atrás apenas da China e Índia. Igualmente, tem-se que em 2013 o Brasil foi responsável pela produção de 43,6 milhões de toneladas de frutas. Dessa produção, estima-se que, aproximadamente, 54\% (23,8 milhões) das frutas foram processadas para a fabricação de sucos, néctares, drinques de frutas e polpas.

Sendo assim, o Brasil tem contribuído significativamente na produção de subprodutos agroindústrias, especialmente de resíduos sólidos. Uma das consequências da produção excessiva desses resíduos consiste no descarte inapropriado, ocasionando problemas ambientas e perdas de biomassa e bioenergia. Dentre as frutas que mais produzem resíduos, destaca-se o abacaxi por conta da posição do Brasil como segundo maior produtor mundial da fruta, conforme dados Food and Agriculture Organization of the United Nations em 2013.

Diversos estudos (Lallo et al.,2003; Selvarajoo \& Hanson, 2014; Wu \& Shiau, 2015; Sah, Vasiljevic, Mckechnie \& Donkor, 2015), na literatura viabilizam a aplicação dos resíduos de abacaxi, como as cascas, na suplementação da alimentação humana e ração animal, na geração de combustíveis renováveis e, em especial, no tratamento de efluentes industriais através da produção de biossorvente.

O uso expressivo de corantes sintéticos para os mais diversos fins, tem gerado grandes volumes de efluentes contaminados por esses compostos orgânicos. A problemática do despejo incorreto de efluentes carregados de corantes nos corpos d'água vai além do aspecto estético uma vez que também afeta o desenvolvimento da vida aquática. A presença de corantes nas águas acomete a penetração da luz solar, promovendo um retardamento no processo de fotossíntese. Além disso, certas classes de corantes, como os azocorantes e seus subprodutos, podem ser carcinogênicos e/ou mutagênico (Kunz, Peralta-Zamora, Moraes \& Durán, 2002).

O tratamento de efluentes contendo corantes é geralmente feito através de métodos tradicionais, como a precipitação química e filtração, tratamento eletroquímico, oxidação ou a redução, troca iônica e evaporação. Contudo, essas tecnologias apresentam alguns inconvenientes, dentre eles os elevados custos, a geração secundária de poluentes e, sobretudo, a ineficiencia na remoção dos diversos tipos de corantes existentes (Hameed, Krishni \& Sata, 2009). A técnica de adsorção surge, então, com um método alternativo que vem sendo bastante estudado em função da sua simplicidade, eficiência na remoção, e redução de custos, 
especialmente quando adsorventes oriundos de biomassa são empregados (Gupta \& Suhas, 2009; Mohammed, Shitu \& Ibrahim, 2014).

O reaproveitamento de resíduos agroindustriais representa, portanto, uma importante estratégia no que tange a diminuição da extração de recursos naturais e na redução do acúmulo de resíduos em lixos e aterros sanitários, além de representar uma solução alternativa e econômica para o tratamento de efluentes contaminados por corantes. Dessa forma, o objetivo deste estudo é avaliar o potencial das cascas do abacaxi (Ananas comosus L. Merril) como adsorvente para remoção de corante têxtil azul de metileno presente em solução aquosa. Para tanto, buscou-se avaliar os efeitos da dose do adsorvente e da concentração inicial do adsorbato na capacidade de adsorção e percentual de remoção. As equações isotérmicas de Langmuir e Freundlich foram aplicadas aos dados experimentais.

\section{REVISÃO BIBLIOGRÁFICA}

\subsection{Abacaxi}

O abacaxizeiro (Ananas comosus L. Merril) consiste em uma espécie oriunda da América do Sul, sendo a mais relevante, no aspecto econômico, da família Bromeliaceae. Pertencente ao gênero Ananas, o abacaxi representa uma fruta de regiões tropicais e subtropicais, a qual apresenta como característica marcante o equilíbrio entre acidez e açúcar, tornando-o muito apreciado para consumo in natura. O fruto é formado da união de 100 a 200 frutilhos (popularmente denominados de "olhos" ou "escamas") dispostos no cilindro central, cujo formato pode ser cilíndrico ou cônico (SEBRAE, 2016).

Mais da metade da produção mundial (59\%) do abacaxi é cultivado por apenas seis países, sendo eles Brasil, Tailândia, Filipinas, China, Índia e Costa Rica (Thé et al, 2010 apud Leonel, Leonel \& Sampaio, 2014). Segundo dados da Food and Agriculture Organization of the United Nations (FAO) e Embrapa, o Brasil ocupou o segundo lugar no ranking mundial de produtores de abacaxi em 2013, apresentando uma produção de 1.655 .887 mil frutos. No âmbito nacional, tem-se que a maior região produtora do fruto é a região nordeste, sendo responsável por $35,21 \%$ do cultivo do abacaxi no país.

A utilização industrial do abacaxi é direcionada para a produção de fruta em calda (fatias ou pedaços), geleias, sucos pasteurizados (concentrado ou não), licor e vinho (SEBRAE, 2016; Nascente, Costa \& Costa, 2005). A elaboração desses produtos, por sua vez, promove a geração de resíduos devido ao não aproveitamento integral do fruto, sendo esses resíduos considerados fontes alternativas de fibras e minerais.

\subsection{Aproveitamento dos resíduos do processamento de abacaxi}

O aproveitamento dos resíduos gerados durante o processamento do abacaxi diminui os danos ambientais causados pelo descarte inadequado dos resíduos, além de agregar valor ao material que até então tinha pouco ou nenhum valor econômico (Viganó, 2012). Nesse sentido, diversas formas de aproveitamento desse resíduo podem ser encontradas na literatura. 
No âmbito da alimentação humana, Marreiro, Amorin e Teixeira (2010), estudaram a elaboração de chá feito com a casca seca em estufa à $60{ }^{\circ} \mathrm{C}$ por 5 horas. $\mathrm{O}$ chá da casca do abacaxi foi bem avaliado na maioria dos aspectos julgados na análise sensorial realizada, representando, portanto, uma fonte de baixo custo de nutrientes essenciais ao corpo, como a vitamina $\mathrm{C}$.

Wu e Shiau (2015), investigaram a extração de fibra da casca de abacaxi e a sua incorporação na elaboração de pães e constaram que pães com até $10 \%$ de substituição de farinha por fibra de casca de abacaxi foram os mais aceitos sensorialmente. Sah et al. (2015), avaliaram a geração de peptídeos antioxidantes e antimutogênicos por Lactobacillus acidophilus em iogurtes suplementados com $1 \%$ de casca de abacaxi seca e os resultados sugerem que o pó pode ser utilizado para conferir efeitos prebióticos e fomentar a liberação de peptídeos bioativos na produção de iogurtes.

Além disso, existem também estudos relatando a utilizando desse resíduo agroindustrial em ração de animais. Lallo et al. (2003), estudaram a degrabilidade efetiva da matéria seca e proteína bruta de quatro rações experimentais, com diferentes níveis de níveis de substituição (20, 40 e 60\%) da silagem de milho pela silagem de resíduos industriais de abacaxi (casca, cilindro central e coroa). Os autores constaram que os níveis de substituição não alteraram significativamente a dinâmica de fermentação ruminal devido ao material ser rico em pectina e que, portanto, a silagem de resíduo de abacaxi consiste em uma alternativa viável para a redução dos custos com ração animal.

Outra área promissora para aplicação das cascas de abacaxi como matéria-prima consiste na produção de combustíveis renováveis. Selvarajoo e Hanson (2014), investigaram as condições ótimas para a obtenção de biocarvão através da pirólise de cascas de abacaxi previamente desidratadas. Os autores observaram que a temperatura ideal para a operação foi à $425{ }^{0} \mathrm{C}$ a fim de que o rendimento ficasse em torno de $39 \%$ de carvão (em base úmida). O poder calorífico associado ao biocarvão gerado foi $26.1 \mathrm{MJ}$ kg -1. García-Rosales et al. (2013), afirmam que a estrutura da casca de abacaxi é composta por $67,88 \%$ de fibras (incluindo celulose, hemicelulose, lignina e sílica), configurando-se como uma boa matéria-prima para a produção de carvão.

Rani e Nand (2004) investigaram a produção de metano através da digestão anaeróbica da casca de abacaxi e observaram que o rendimento do biogás foi de $0,67 \mathrm{~m} 3 / \mathrm{kg}$ de sólidos, sendo $65 \%$ do biogás constituído por metano. Similarmente, bioetanol também pode ser produzido, via fermentação por Saccharomyces cerevisiae, utilizando as cascas de abacaxi como substrato, conforme reportado por Ruangviriyachai, Niwaswong, Kosaikanon, Chanthai e Chaimart (2010).

As cascas de abacaxi também podem utilizadas no tratamento de efluentes contaminados por corantes sintéticos ou metais pesados. Dacera, Babel e Parkpian (2009) utilizaram cascas de abacaxi para produzir, pela ação fermentativa do Aspergillus nige, líquido rico em ácido cítrico, o qual foi empregado na lixiviação de íons metálicos de cádmio, cobre, cromo, chumpo, níquel e zinco. Por fim, outros exemplos de utilização das cascas de abacaxi consistem na produção de hidrogel (Hu, Hu, Zeng, Zhao \& Huang, 2010) e extração de bromelina (Ketnawa, Rawdkuen \& Chaiwut, 2010). 


\subsection{Adsorção}

A adsorção ocorre através da difusão de partículas presentes em uma fase fluida para um sólido poroso devido à existência de forças atrativas que emanam da superfície do sólido. Nesse contexto, denomina-se como adsorvente o sólido no qual ocorre o fenômeno e adsorbato o soluto o que será adsorvido. A retenção dessas partículas à matriz sólida é feita por intermédio de ligações químicas ou forças intermoleculares. Sendo assim, de acordo com a natureza dessa interação adsorvato-adsorvente, a adsorção é classificada em duas categorias: adsorção física (fisissorção) e adsorção química (quimissorção) (Seader, Henley \& Hoper, 2010; Coulson \& Richardson, 2002).

$\mathrm{Na}$ adsorção física ou fisissorção, observa-se a existência de forças intermoleculares, especialmente de Van der Waals, entre o adsorvato e o adsorvente. As interações de Van der Waals apresentam um longo alcance, contudo são fracas. Dessa forma, fisissorção tem como principal característica a reversibilidade do processo, apresentando valores de entalpia em torno de $20 \mathrm{~kJ} \mathrm{~mol}^{-1}$ (Atkins, 2006). Além disso, nesse tipo de adsorção, pode ocorrer a sobreposição de camadas de adsorbato sobre a superfície do sólido, diminuindo, assim, a capacidade adsortiva à medida que o número de camada cresce. (Coulson \& Richardson, 2002).

Já na adsorção química ou quimissorção, o adsorbato liga-se à superfície do adsorvente através de ligações químicas, geralmente do tipo iônica ou covalente, as quais são significativamente mais intensas quando comparadas com as interações relacionadas à fisissorção. Por conta disso, a entalpia da quimissorção é muito maior do que a de fisissorção, apresentando valores a partir de $200 \mathrm{kJmol}^{-1}$ (Atkins, 2006).

Diferente do que ocorre na fisissorção, a quimissorção apresenta a formação de uma monocamada de adsorbato sob a superfície do sólido e as forças de adsorção decrescem conforme a extensão da superfície ocupada do sólido aumenta devido à interação entre as partículas de adsorbato. É importante destacar, no entanto, que após a formação da monocamada na quimossorção, pode haver a sob posição de uma outra camada de adsorbato que foi adsorvido fisicamente (Coulson \& Richardson, 2002).

O grau de adsorção de um soluto sob uma superfície porosa é controlado por diversos fatores tais como temperatura, estrutura do adsorvente e agitação. Segundo Cooney (1999 apud Curbelo, 2002) o efeito da temperatura, em geral, consiste na redução da capacidade de adsorção com a elevação da temperatura, o que se deve, em parte, ao fato de que as partículas adsorvidas passam a apresentar maiores energias vibracionais, provocando, assim, a dessorção do adsorbato da superfície do sólido. Contudo, deve-se considerar que alguns sistemas podem ter esta capacidade elevada com o aumento da temperatura.

Já quanto à estrutura do adsorvente, Coulson e Richardson (2002) afirmam que um adsorvente é considerado comercialmente atraente quando esse apresenta características como extensa área superficial interna, a qual deve ser acessível através de poros grandes o suficiente para admitir a adsorção das partículas de interesse. Por fim, a velocidade de agitação influencia o fenômeno de adsorção na medida que um aumento do grau de agitação promove um aumento na turbulência e diminuição da espessura da camada limite, favorecendo assim, a transferência de massa da solução para o adsorvente (Patil, Renukdas \& Patel, 2012). 
A determinação da relação existente entre a concentração do adsorbato no fluido e na superfície do adsorvente quando o sistema atinge o equilíbrio é dada através das curvas denominadas isotermas de adsorção. A concentração do adsorbato no sólido é dada em função da massa adsorvida por unidade de massa de adsorvente utilizada. (Mccabe, Smith \& Harriot, 1993).

A capacidade de adsorção de dado adsorvente quando em contato com certo adsorbato envolve a interação de três propriedades: a concentração $C$ do adsorbato na fase fluida, a concentração $Q$ do adsorbato na fase sólida e a temperatura do sistema. Se a temperatura é mantida constante, as outras duas variáveis podem ser representadas graficamente, através de curvas denominadas isotermas de adsorção conforme a Figura 1, a fim de que a relação de equilíbrio existente entre as variáveis seja determinada (Coulson \& Richardson, 2002; McCabe, Smith \& Harriott, 1993).

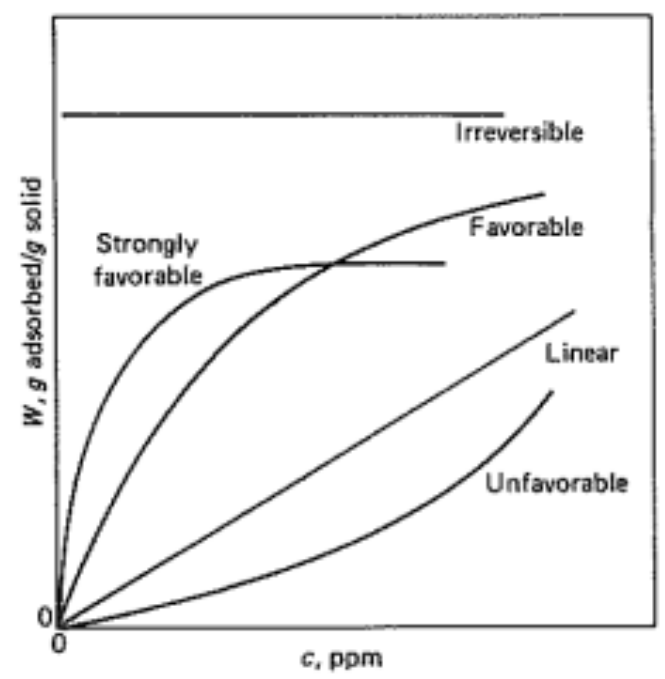

Figura 1: Tipos de isoterma de adsorção. Fonte: Mccabe et al. (1993).

A modelagem matemática das isotermas de adsorção pode ser feita através das equações de Langmuir ou de Freundlich, as quais representam os modelos mais comumente utilizados. Segundo Atkins (2006) e Seader et al., (2010) a isoterma de Langmuir Equação (1) se baseia nas seguintes suposições:

- $\quad$ Ocorre apenas adsorção monomolecular, ou seja, cada sítio pode abrigar apenas uma única molécula (cobertura monocamada);

- $\quad$ A adsorção é localizada (as moléculas são adsorvidas em um número fixo de sítios de localização bem definida);

- A energia de adsorção (a força de ligação gerada entre a superfície e as moléculas de adsorbato) é igual para todos os sítios, ou seja, todos os sítios são energicamente equivalentes;

- $\quad$ Não há interação entre as moléculas adsorvidas em sítios vizinhos.

$$
q_{e}=\frac{K_{L} q_{\max } C_{e}}{\left(1+K_{L} C_{e}\right)}
$$


Sendo:

$q_{e}$ : Concentração de equilíbrio do adsorbato na fase sólida $(\mathrm{mg} / \mathrm{g})$;

$q_{\max }$ : Concentração máxima de adsorbato na superfície do adsorvente requerida para formação de uma monocamada (mg/g);

$K_{L}$ : Constante de equilíbrio de Langmuir (L/mg);

$C_{e}$ : Concentração de equilíbrio do adsorbato na fase fluida $(\mathrm{mg} / \mathrm{L})$.

Hameed et al. (2009), afirmam que a avaliação da forma da isoterma de Langmuir pode ser feita através do cálculo do fator de separação Equação (2), conforme os critérios listados na Tabela 2.

$$
R_{L}=\frac{1}{\left(1+K_{L} C_{0}\right)}
$$

Sendo:

$R_{L}$ : Fator de separação;

$K_{L}$ : Constante de equilíbrio de Langmuir (L/mg);

$C_{0}$ : Concentração inicial máxima de adsorbato $(\mathrm{mg} / \mathrm{L})$.

Tabela 2: Forma da isoterma de Langmuir de acordo com o fator de separação (R)

$\begin{array}{cc}\text { Valor do fator }\left(R_{L}\right) & \text { Tipo de isoterma } \\ R_{L}>1 & \text { Desfavorável } \\ 0<R_{L}<1 & \text { Favorável } \\ R_{L}=0 & \text { Irreversível } \\ R_{L}=1 & \text { Linear }\end{array}$

Nessa mesma perspectiva, um outro modelo aplicado na descrição matemática do fenômeno de adsorção foi proposto por Freundlich (1907). Curbelo (2002), assegura que a isoterma de Freundlich, dada pela Equação (3), representa uma das primeiras equações empíricas propostas para estabelecer uma relação entre a quantidade de material adsorvido e a concentração do material na solução. O modelo considera a superfície do adsorvente como sendo heterogênea e com distribuição não uniforme do calor de adsorção, o que implica na ocupação inicial dos sítios energicamente mais favoráveis (Atkins, 2006).

$$
q_{e}=K_{L} C_{e}^{\frac{1}{n}}
$$

Sendo:

$q_{e}$ : Concentração de equilíbrio do adsorbato na fase sólida $\left(\mathrm{mg} \mathrm{g}^{-1}\right)$;

$K_{L}$ : Constante de equilíbrio de Freundlich;

$C_{e}$ : Concentração de equilíbrio do adsorbato na fase fluida ( $\mathrm{mg} \mathrm{L}^{-1}$ )

$n$ : Constante associada ao modelo. 
O modelo de Freundlich é, frequentemente, a melhor representação teórica para os dados obtidos experimentalmente para adsorção em fase líquida. Em geral, para esse modelo, o aumento da temperatura do sistema promove a diminuição do valor da constante de equilíbrio KF e o aumento do valor de $n$ (Seader et al., 2010; Mccabe et al., 1993).

\section{METOdOLOGIA}

\subsection{Materiais}

As cascas de abacaxi (Ananas comosus) foram fornecidas por uma unidade de processamento de polpas de fruta no município do Natal-RN. O azul de metileno (AM) foi utilizado como adsorbato modelo para os ensaios de adsorção por conta da importância desse corante catiônico para as indústrias têxtil e farmacêutica. O AM consiste em um composto orgânico aromático heterocíclico, inodoro e solúvel em água. Sua fórmula molecular é dada por $\mathrm{C}_{16} \mathrm{H}_{18} \mathrm{CIN}_{3} \mathrm{~S}$ com massa molar de 319,86g/mol (Bruno, 2008). A estrutura química do azul de metileno é representada pela Figura 2. As propriedades físico-químicas e especificações desse corante estão sumarizadas na Tabela 3.

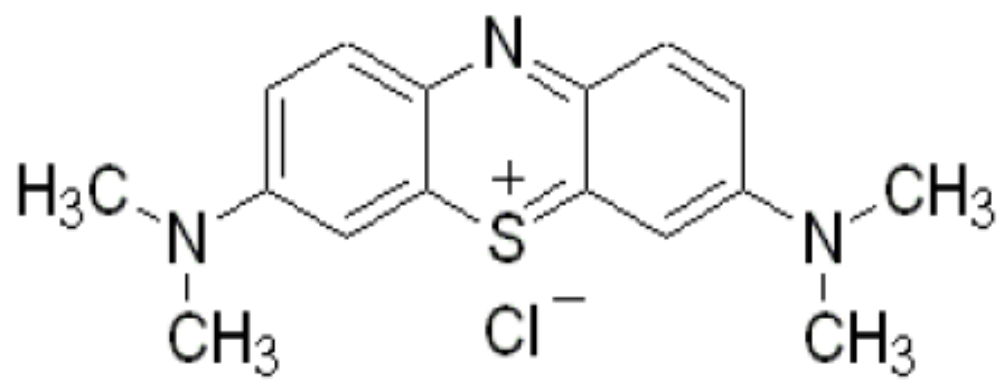

Figura 2: - Estrutura química do corante azul de metileno. Fonte: Bruno (2008).

Tabela 3: Especificações do corante azul de metileno (AM). Fonte: Merk chemicals.

\begin{tabular}{cc}
\hline Ponto de fusão & $180^{\circ} \mathrm{C}($ decomposição $)$ \\
$\mathrm{pH}$ & $3\left(10 \mathrm{~g} / \mathrm{L}, \mathrm{H}_{2} \mathrm{O}, 20^{\circ} \mathrm{C}\right)$ \\
Densidade & $400-600 \mathrm{~kg} / \mathrm{m}^{3}$ \\
Solubilidade & $50 \mathrm{~g} / \mathrm{L}\left(20^{\circ} \mathrm{C}\right)$ \\
$\Lambda$ de absorbância máxima & $660-665 \mathrm{~nm}$ \\
\hline
\end{tabular}

\subsection{Ensaio de adsorção}

\subsubsection{Preparo do adsorvente}

As cascas de abacaxi foram secadas à $80^{\circ} \mathrm{C}$ e posteriormente trituradas, obtendo-se um pó com granulometria menor que $0,2 \mathrm{~mm}$.

\subsubsection{Efeito da massa de adsorvente}

A influência da massa de adsorvente da casca de abacaxi no percentual de remoção do corante foi avaliada através de 5 ensaios (tabela 4). Os experimentos foram conduzidos à $30^{\circ} \mathrm{C}$, 
sob rotação de $150 \mathrm{rpm}$ e utilizando $50 \mathrm{~mL}$ de solução aquosa de azul de metileno (100 $\mathrm{mg} \mathrm{L}^{-1}$ ) por 240 minutos. Após esta etapa, as amostras foram centrifugadas por 20 minutos a $3000 \mathrm{rpm}$ e o líquido sobrenadante foi coletado para ser analisado através de espectrofotometria UV/Visível. 0 percentual de adsorção foi calculado pela Equação (4).

$$
\% R=\frac{\left(C_{0}-C\right)}{C_{0}} \times 100
$$

Tabela 4: Condições operacionais dos ensaios de avaliação da massa de adsorvente

\begin{tabular}{cc}
\hline Experimento & Massa Adsorvente (g) \\
\hline 1 & 0,1 \\
2 & 0,2 \\
3 & 0,3 \\
4 & 0,4 \\
5 & 0,5 \\
\hline
\end{tabular}

\subsection{Metodologia Analítica}

A concentração de azul de metileno na fase aquosa após a adsorção foi determinada através de espectrofotometria UV/Visível, utilizando o espectrofotômetro Biospectro ${ }^{\circledR}$ (modelo SP-220) no comprimento de onda $660 \mathrm{~nm}$. Soluções padrão de azul de metileno (concentração de 2 ppm a 10 ppm) foram utilizadas para construir a curva de calibração do soluto em questão, baseando-se na lei de Lambert Beer.

\subsubsection{Isoterma de adsorção}

O procedimento experimental para obter as isotermas de adsorção consistiu em realizar os ensaios de adsorção com uma massa constante de adsorvente $(0,5 \mathrm{~g})$ em contato com $50 \mathrm{~mL}$ de solução de azul de metileno a diferentes concentrações (Tabela 5). As amostras foram mantidas sob agitação de $150 \mathrm{rpm}$ por 240 minutos. Esses ensaios foram conduzidos em duas condições de temperatura ( $30 \stackrel{\circ}{\circ}$ e $40 \stackrel{\circ}{\circ}$ ) afim de avaliar o efeito da temperatura na distribuição do soluto entre as fases aquosa e sólida.

Tabela 5: Condições operacionais dos ensaios de avaliação da isoterma de adsorção

\begin{tabular}{cc}
\hline Experimento & $\begin{array}{c}\text { Concentração da solução de AM } \\
\left(\mathbf{m g ~ L}^{-\mathbf{1}}\right)\end{array}$ \\
\hline 1 & 60 \\
2 & 70 \\
3 & 80 \\
4 & 90 \\
5 & 100 \\
\hline
\end{tabular}

Conforme já descrito, a concentração do azul de metileno na fase aquosa foi determinada por espectrofotometria UV/Visível. Já a concentração do corante na fase sólida foi determinada através de balanço de massa, expresso pela Equação (5). Por fim, os modelos de isotermas de adsorção proposto por Langmuir e Freundlich foram utilizados na descrição matemática dos resultados experimentais obtidos. 


$$
q_{e}=\frac{\left(C_{0}-C_{e}\right) V}{m_{a}}
$$

Sendo, $\left(\mathrm{mg} \mathrm{g}^{-1}\right)$;

$q_{e}=$ Quantidade de soluto adsorvido por unidade de massa de adsorvente, no equilíbrio

$C_{0}=$ Concentração inicial da solução (ppm);

$C_{e}=$ Concentração da solução no equilíbrio (ppm);

$V=$ Volume da solução (L);

$m_{a}=$ Massa do adsorvente $(\mathrm{g})$

\section{RESULTADOS E DISCUSSÃO}

\subsection{Curva de calibração do azul de metileno}

Os valores de absorbância para cada uma das soluções padrão de azul de metileno analisadas são mostrados na figura 3. Os resultados obtidos são similares aos encontrados por Tayeb e Hussein (2015). A regressão linear dos dados experimentais apresentou um coeficiente de determinação satisfatório $\left(R^{2}=0,9987\right)$, o que valida, portanto, a expressão matemática obtida para representar a relação entre a absorbância e a concentração do soluto. Nesse sentido, a equação da reta será utilizada na determinação da concentração de azul de metileno na fase aquosa.

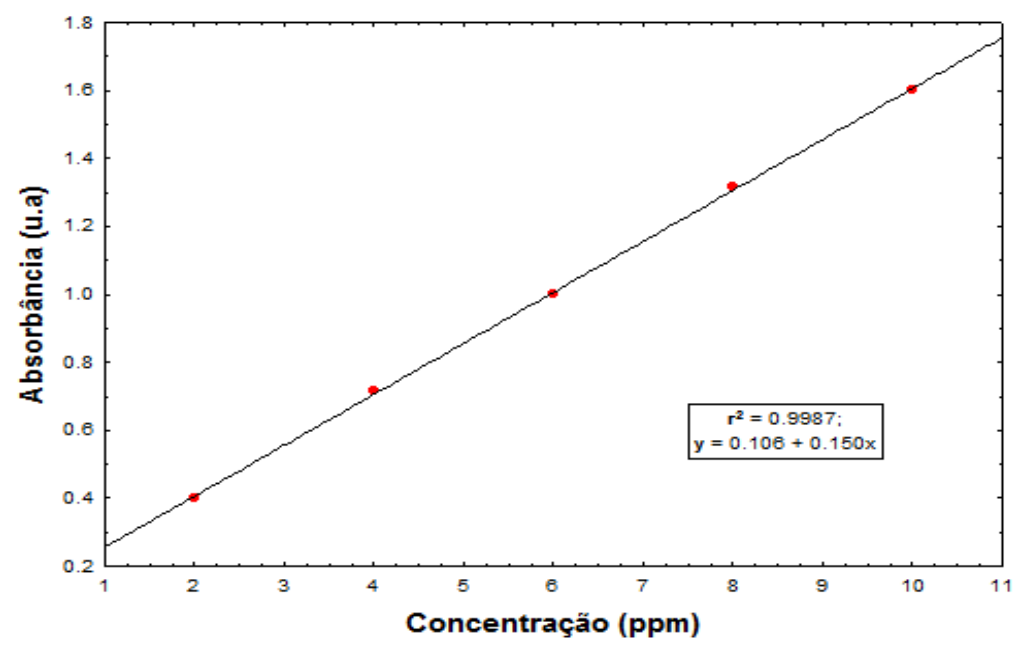

Figura 3: Curva de calibração para azul de metileno

\subsection{Efeito da massa de adsorvente da casca de abacaxi}

A figura 4 mostra que a relação entre a massa de adsorvente e o percentual de remoção (\%R) é diretamente proporcional, enquanto que o efeito da massa de adsorvente na concentração de azul de metileno na superfície do adsorvente $\left(q_{e}\right)$ foi inversamente proporcional. Em outras 
palavras, a remoção do corante foi favorecida quando a massa de adsorvente empregada aumentou de $0.1 \mathrm{~g}$ (38,30\% de remoção) para $0.5 \mathrm{~g}$ (77,25\% de remoção). Igualmente, tem-se que a eficiência de remoção do adsorvente, expressa através $q_{e}$, diminuiu com o aumento da massa de adsorvente. A literatura é vasta de trabalhos que apresentam esse mesmo comportamento para ensaios de adsorção de corantes por biossorventes.

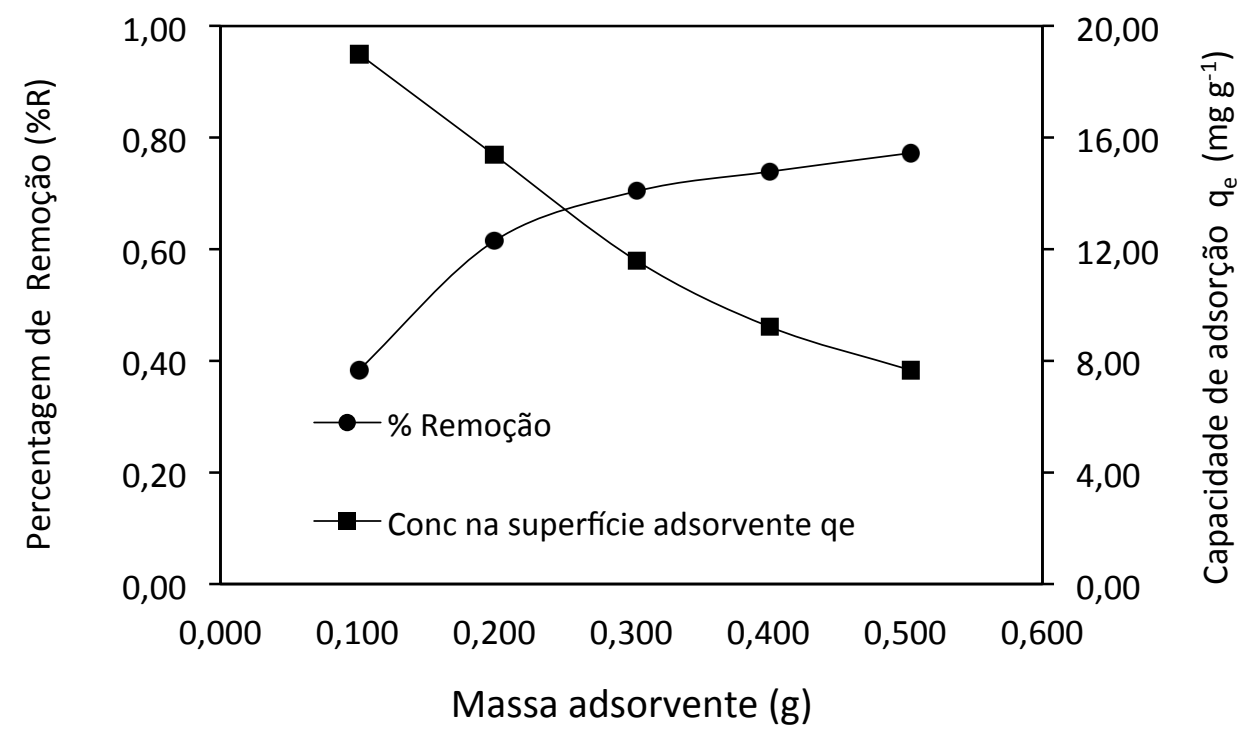

Figura Error! No text of specified style in document.: Efeito da massa de adsorvente empregada na adsorção de azul de metileno

Ponnusami, Vikram e Srivastava (2008), realizaram ensaios de adsorção de azul de metileno em pó de folha da goiaba e observaram que o aumento da massa de adsorvente (incremento de 0,5 g para $2 \mathrm{~g}$ ) aumentou o percentual de remoção do adsorbato de 62,1 \% para 97,89\%. Igualmente, Uddin, Islam, Mahmud e Rukanuzzaman (2009), avaliaram a remoção de azul de metileno através de resíduos de chá com granulometria na faixa de 180-300 $\mu \mathrm{m}$ e também constataram que o percentual de remoção e a concentração de adsorbato na superfície do adsorvente $\left(\mathrm{q}_{\mathrm{e}}\right)$ sofreram influência da dose de adsorvente. Outros trabalhos utilizando adsorventes oriundos de biomassa, como casca de castanha de caju (Subramaniam \& Ponnusamy, 2015) e casca de coco (Fo \& Odebunmi, 2010) observaram esse mesmo comportamento, confirmando, portanto, a existência do efeito da dosagem de adsorvente sob as variáveis avaliadas.

A explicação para o favorecimento do percentual de remoção é atribuída ao fato de que o incremento da dose de adsorvente aumenta a área de adsorção por conta do maior número de sítios disponíveis. Além disso, o aumento da massa também faz com que alguns sítios presentes na superfície do adsorvente não sejam ocupados por não serem energeticamente favoráveis, diminuindo assim o valor de $q_{e}$ (Reddy, Verma \& Subrahmanyam, 2016).

\subsection{Efeito da concentração inicial do azul de metileno}

O aumento da concentração inicial do corante implicou na redução do percentual de remoção (R\%) para as duas temperaturas estudadas, conforme mostra a figura 5. Esse comportamento é atribuído à saturação da superfície do adsorvente, o que dificulta a adsorção do corante em camadas interiores do sólido. Reddy et al (2016) relataram redução de aproximadamente $12 \%$ na porcentagem de remoção ao aumentarem a concentração inicial da 
solução de azul de metileno de $100 \mathrm{mg} \mathrm{L}^{-1}$ para $200 \mathrm{mg} \mathrm{L}^{-1}$ em ensaios de adsorção utilizando sabugo de milho.

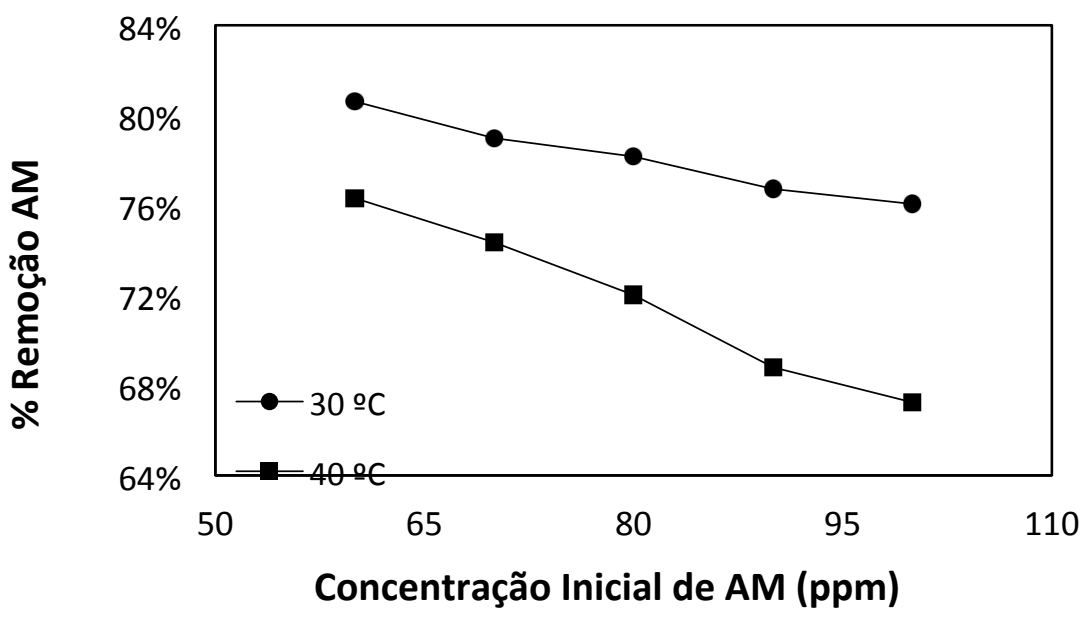

Figura 5: Efeito da concentração inicial de adsorbato sob o percentual de remoção

A figura 6 mostra que o aumento da concentração inicial de AM tornou mais eficiente a remoção do azul de metileno da fase aquosa, ou seja, aumentou a capacidade de adsorção $\left(q_{\mathrm{e}}\right)$. $A$ explicação para esse resultado consiste no fato de que quanto maior for a concentração inicial do adsorbato, maior será a força motriz (gradiente de concentração) responsável pelo transporte do corante da fase aquosa para a fase sólida. Senthil et al. (2014) observou o mesmo comportamento para experimentos similares, utilizando casca de laranja tratada com ácido sulfúrico como adsorvente de azul de metileno.

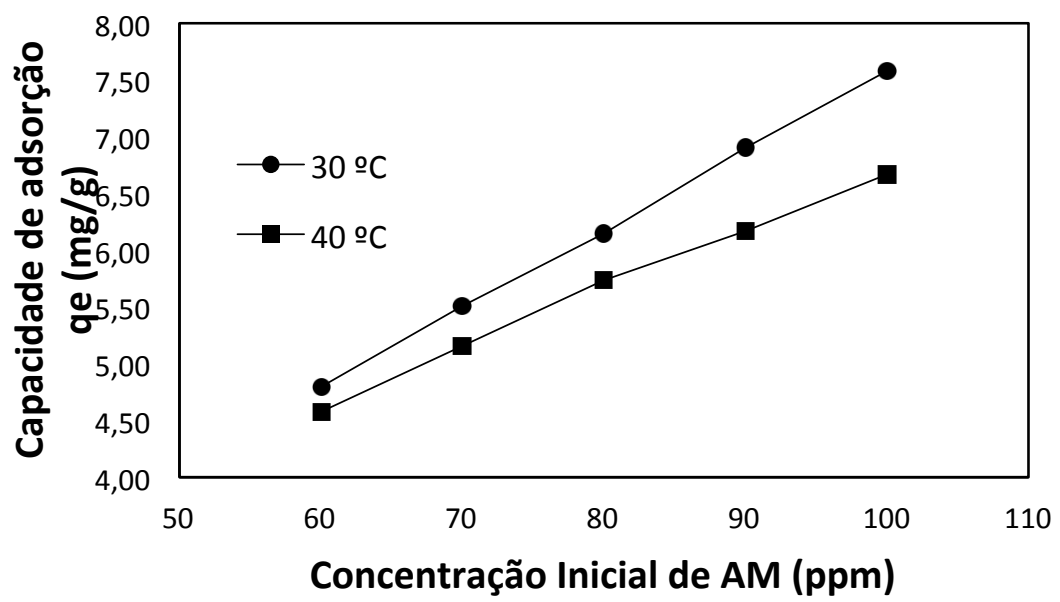

Figura 6: Efeito da concentração inicial de adsorbato sob a capacidade de adsorção (qe)

\subsection{Isotermas de adsorção e modelagem matemática}

As isotermas de adsorção consistem em uma forma gráfica de representar a distribuição do adsorbato entre a fase sólida $\left(\mathrm{q}_{\mathrm{e}}\right)$ e a fase líquida $\left(\mathrm{C}_{\mathrm{e}}\right)$ do sistema à temperatura constante. Os modelos de isoterma permitem a determinação da capacidade máxima de adsorção ( $q_{\mathrm{emáx}}$ ) de certo adsorvente, assumindo a formação de uma monocamada saturada de moléculas de soluto 
na superfície do adsorbato (Hameed et al., 2009). Sendo assim, os modelos de Langmuir e Freunlich foram utilizados para descrever os dados de equilíbrio obtidos à 30 e $40^{\circ} \mathrm{C}$ (Figura 7).

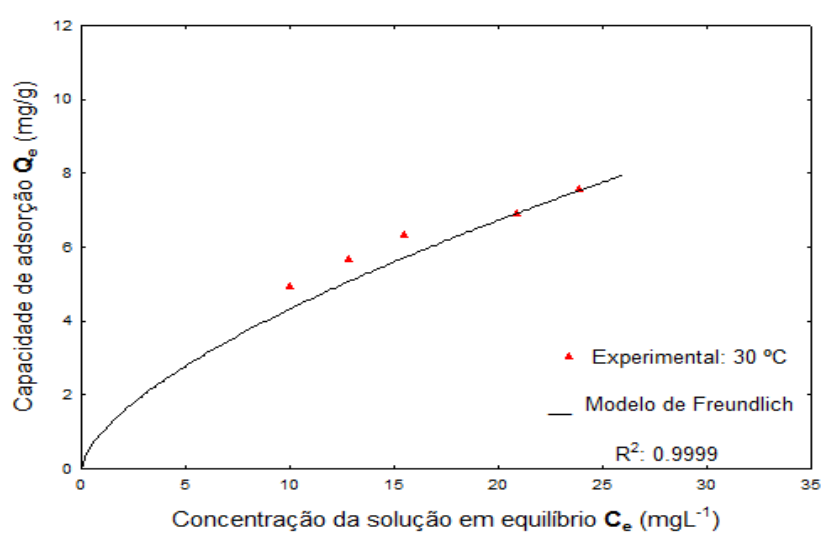

(a)

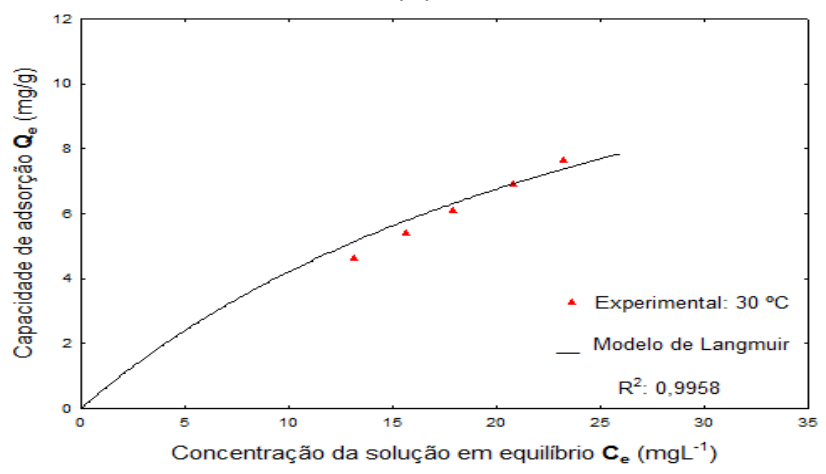

(c)

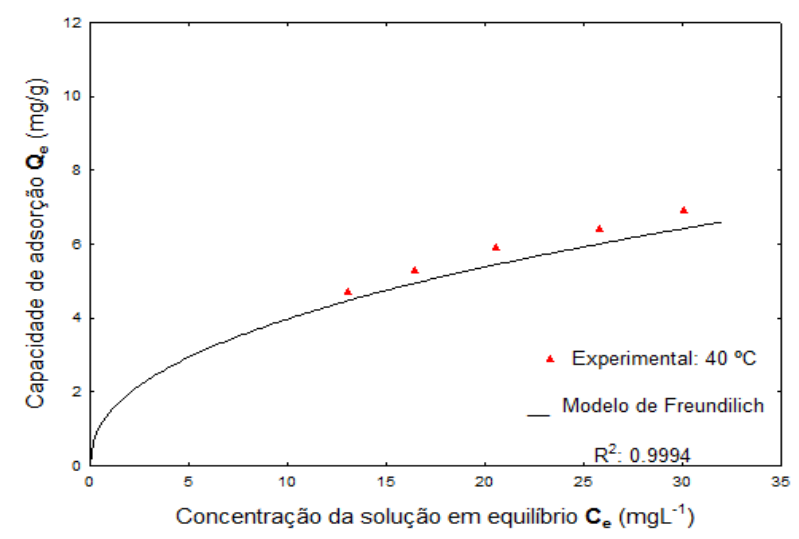

(b)

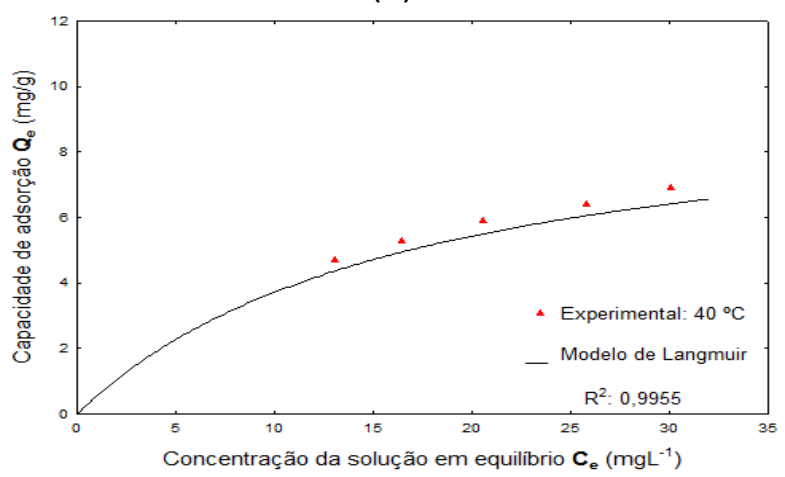

(d)

Figura 7: Isotermas de adsorção descritas pelos modelos de Freundlich à $30^{\circ} \mathrm{C}$ (a) e $40^{\circ} \mathrm{C}(\mathrm{b})$ e de Langmuir à $30^{\circ} \mathrm{C}$ (c) e $40^{\circ} \mathrm{C}$ (d)

Os parâmetros obtidos a partir do ajuste de cada modelo são mostrados na Tabela 6, bem como o valor do fator de separação $\left(R_{L}\right)$, o qual avalia o quão favorável é o processo de adsorção nas condições estudadas.

Tabela 6: Parâmetros associados aos modelos de isoterma de Langmuir e Freundlich

\begin{tabular}{cccc}
\hline \multirow{2}{*}{ Isotermas de adsorção } & Parâmetros & \multicolumn{2}{c}{ Temperatura } \\
& $\mathbf{3 0}^{\circ} \mathbf{C}$ & $\mathbf{4 0} \mathbf{C}$ \\
\hline \multirow{2}{*}{ Langmuir } & $\mathrm{K}_{\mathrm{L}}\left(\mathrm{L} \mathrm{mg}^{-1}\right)$ & 0,0326 & 0,05858 \\
& $\mathrm{Q}_{\mathrm{emáx}}\left(\mathrm{mg} \mathrm{g}^{-1}\right)$ & 17,1179 & 10,0616 \\
& $\mathrm{R}^{2}$ & 0,9958 & 0,9954 \\
\multirow{3}{*}{ Freundlich } & $\mathrm{R}_{\mathrm{L}}$ & 0,2347 & 0,1458 \\
& $\mathrm{~K}_{\mathrm{F}}$ & 0,9952 & 1,4562 \\
& $\mathrm{n}$ & 1,5677 & 2,2934 \\
& $\mathrm{R}^{2}$ & 0,9999 & 0,9994 \\
\hline
\end{tabular}

Observa-se que o modelo de Freundlich representou mais eficientemente aos dados experimentais $\left(R^{2}=0,9999\right.$ e 0,9994 para as temperaturas de 30 e $\left.40^{\circ} \mathrm{C}\right)$. No entanto, por se tratar de um modelo empírico, o modelo de Langmuir é preferencialmente utilizado para descrição de dados experimentais de adsorção. $O$ valor da capacidade máxima de adsorção ( $q_{\text {emáx }}$ ) calculado foi de $17,1179 \mathrm{mg} \mathrm{g}^{-1}$ e $10,0616 \mathrm{mg} \mathrm{g}^{-1}$ a 30 e $40{ }^{\circ} \mathrm{C}$, respectivamente. Os resultados indicam, portanto, que a capacidade de adsorção aumentou com a diminuição da temperatura, sugerindo 
que a reação de adsorção tem caráter exotérmico. Além disso, o valor encontrado para o fator de separação $R_{L}$ sugere que a adsorção é favorável nas condições estudas.

A comparação dos resultados obtidos nesse trabalho com outros estudos envolvendo o emprego de resíduos agroindustriais como adsorvente de corante têxtil (tabela 7) demostram o potencial da casca de abacaxi como adsorvente. Uma forma de aperfeiçoar o uso desse resíduo como adsorvente é através do ajuste do pH de operação. Lupti, Shian e Kamarudzaman (2011) obteve $q_{\text {máx }}$ igual a $78,125 \mathrm{mg} \mathrm{g}^{-1}$ em ensaios de adsorção, utilizando cascas de abacaxi, semelhantes aos realizados neste trabalho, diferindo apenas o $\mathrm{pH}$ do sistema, o qual foi ajustado para 12. Uma outra forma de otimizar a capacidade de adsorção da casca de abacaxi é através de tratamento térmico. Foo e Hammed (2012) utilizaram cascas de abacaxi na produção de carvão ativado assistido por microondas e utilizando $\mathrm{KOH}$ e $\mathrm{K}_{2} \mathrm{CO}_{3}$ no processo de ativação. $\mathrm{O}$ ensaio de adsorção de azul de metileno pelo carvão produzido apresentou capacidade de adsorção máxima de $462.10 \mathrm{mg} / \mathrm{g}$.

Tabela 7: Capacidade de adsorção do azul de metileno por outros adsorventes oriundos de resíduo agroindustrial

\begin{tabular}{lcccc}
\hline \multicolumn{1}{c}{ Adsorvente } & Condições & Granulometria & Qmáx (mg/g) & Referência \\
\hline Casca de trigo & $30 \circ \mathrm{C} \mathrm{pH=9}$ & $0,6 \mathrm{~mm}$ & 16,56 & Bulut e Aydin (2006) \\
Resíduo de palmito & $30 \circ \mathrm{C} \mathrm{pH=7}$ & $42 \mathrm{mesh}$ & 50,90 & Honorato et al (2015) \\
Casca de laranja/banana & $30 \circ \mathrm{C} \mathrm{pH=7}$ & $<0,5 \mathrm{~mm}$ & $18,60 / 20,80$ & Annadurai et al (2002) \\
Casca de abacaxi & $30 \circ \mathrm{C}$ & $<0,2 \mathrm{~mm}$ & 17,12 & Presente trabalho \\
Casca de maracujá & $25 \circ \mathrm{C} \mathrm{pH=9}$ & $0,2 \mathrm{~mm}$ & 2,17 & Pavan et al (2008) \\
\hline
\end{tabular}

\section{CONCLUSÃO}

O adsorvente obtido a partir de casca de abacaxi seca apresentou resultados satisfatórios, com remoção de até $81 \%$ do azul de metileno presente em solução aquosa. Com o estudo das variáveis operacionais observou-se que o aumento da massa de adsorvente favoreceu a remoção do corante. Já o aumento da concentração inicial do corante, mantendo-se a massa de adsorvente fixa, provocou uma redução do percentual de remoção.

Quanto a capacidade de adsorção os resultados mostraram que foi favorecida pelo aumento da concentração do azul de metileno. Observou-se também que a separação apresentou melhores resultados quando conduzida a temperaturas mais baixas, sugerindo, assim, o caráter exotérmico da reação. Os dados experimentais foram melhor representados pelo modelo de Langmuir, com valores da capacidade de adsorção máxima ( $q_{\text {emáx }}$ ) iguais a $17,1179 \mathrm{mg} \mathrm{g}^{-1}$ e 10 , $0616 \mathrm{mg} \mathrm{g}^{-1}$ a 30 e 40 o $\mathrm{C}$, respectivamente.

O aproveitamento das cascas de abacaxi, portanto, representa uma forma de reduzir o acúmulo de resíduos em lixos e aterros sanitários e uma alternativa econômica e viável para solucionar a problemática envolvendo efluentes contaminados por corantes têxteis. 


\section{REFERÊNCIAS}

Annadurai, G., Juang, R.S., Lee, D.J. (2002). Use of cellulose based wastes for adsorption of dyes from aqueous solutions. J. Hazard. Mater. B29, 263-274.

Atkins, P. W. (2006). Physical chemistry. Oxford [etc.]: Oxford University Press.

Bruno, M. (2008). Utilização de zeólitas sintetizadas a partir de cinzas de carvão na remoção de corante em água (Doctoral dissertation, Universidade de São Paulo).

Bulut, Y. e Aydin, H. (2006). A Kinetics and Thermodynamics Study of Methylene Blue Adsorption on Wheat Shells. Desalination, 194, 259-267

Cooney, D. O. (1998). Adsorption design for wastewater treatment. CRC Press.

Coulson, J. M., \& Richardson, J. F. (2002). Chemical Engineering-Particle Technology and Separation Process, vol. 2.

Curbelo, F. D. S. (2002). Estudo da remoção de óleo em águas produzidas na indústria de petróleo, por adsorção em coluna utilizando a vermiculita expandida e hidrofobizada. Natal, Universidade Federal do Rio Grande do Norte-UFRN, Tese de Mestrado.

Dacera, D.; Babel, S.; Parkpian, P. (2009). Potential for land application of contaminated sewage sludge treated with fermented liquid from pineapple wastes. Journal of hazardous materials, 167(1), p.866-872.

Fo, O., \& Odebunmi, E. O. (2010). Freundlich and Langmuir isotherms parameters for adsorption of methylene blue by activated carbon derived from agrowastes. Advances in Natural and applied Sciences, 4, 281-288.

FOOD AND AGRICULTURE ORGANIZATION - FAOSTAT. (2013). Disponível em: <http://faostat.fao.org>. Acesso em: 13 fev. 2017.

Foo, K. Y., \& Hameed, B. H. (2012). Coconut husk derived activated carbon via microwave induced activation: effects of activation agents, preparation parameters and adsorption performance. Chemical Engineering Journal, 184, 57-65.

García-Rosales, G., Longoria-Gándara, L. C., Martínez-Gallegos, S., \& González-Juárez, J. (2013). Synthesis and Characterization of Carbon Conditioned with Iron Nanoparticles Using Pineapple-Peel. Advances in Nanoparticles, 2, 384-390.

Gupta, V. K. (2009). Application of low-cost adsorbents for dye removal-A review. Journal of environmental management, 90(8), 2313-2342.

Hameed, B., Krishni, R. R., \& Sata, S. A. (2009). A novel agricultural waste adsorbent for the removal of cationic dye from aqueous solutions. Journal of hazardous materials, 162(1), 305311.

Haque, E., Lo, V., Minett, A.I., Harris, A.T. and Church, T.L. (2014). Dichotomous adsorption behaviour of dyes on an amino-functionalised metal-organic framework, amino-MIL-101 (Al). Journal of Materials Chemistry A, 2(1), 193-203.

Honorato, A.C., Machado, J.M., Celante, G., Borges, W.G., Dragunski, D.C. E Caetano, J. (2015). Biossorção de azul de metileno utilizando resíduos agroindustriais. Revista Brasileira de Engenharia Agrícola e Ambiental, 19(7), pp.705-710. 
Hu, X., Hu, K., Zeng, L., Zhao, M., \& Huang, H. (2010). Hydrogels prepared from pineapple peel cellulose using ionic liquid and their characterization and primary sodium salicylate release study. Carbohydrate Polymers, 82(1), 62-68.

Ketnawa, S.; Rawdkuen, S.; Chaiwut, P. (2010).Two phase partitioning and collagen hydrolysis of bromelain from pineapple peel Nang Lae cultivar. Biochemical Engineering Journal, 52(2), p.205-211.

Kunz, A., Peralta-Zamora, P., de Moraes, S. G., \& Durán, N. (2002). Novas tendências no tratamento de efluentes têxteis. Química nova, 25(1), 78-82.

Lallo, F. H., Prado, I. N., Nascimento, W. G., Zeoula, L. M., Moreira, F. B., Wada, F. Y. (2003). Níveis de substitução da silagem de milho pela silagem de resíduos industriais de abacaxi sobre a degradabilidade ruminal em bovinos de corte. R. Bras. Zootec, 32(3), p.719-726.

Leonel, S., Leonel, M., \& Sampaio, A. C. (2014). Processamento de frutos de abacaxizeiro cv smooth cayenne: perfil de açúcares e ácidos dos sucos e composição nutricional da farinha de cascas. Revista Brasileira de Fruticultura, 433-439.

Lupti, N. A., Shian, W. Y., \& Kamarudzaman, A. N. (2011). Removal of methylene blue using pineapple peel powder as adsorbent. In Proceedings of the 3rd CUTSE International Conference (pp. 352-356).

Marreiro, A.; Amorin, M.; Teixeira, P. (2010). Elaboração do chá da casca do abacaxi (Ananas comosus-bromeliaceae) e caracterização das propriedades fisico-químicas e sensoriais. Revista Acta Tecnológica, 5(1), p.82-90.

McCabe, W. L., Smith, J. C., \& Harriott, P. (1993). Unit operations of chemical engineering (Vol. 5, p. 154). New York: McGraw-Hill.

Mohammed, M. A., Shitu, A., \& Ibrahim, A. (2014). Removal of methylene blue using low cost adsorbent: a review. Research Journal of Chemical Sciences ISSN, 2231, 606X.

Moretti, C. L. (2006). Aproveitamento de resíduos do processamento mínimo de frutas e hortaliças. IV Encontro Nacional sobre Processamento Mínimo de Frutas e Hortaliças, I Simpósio Íbero Americano de vegetais frescos cortados.

Nascente, A. S., Da Costa, R. S. C., \& Costa, J. N. M. (2005). Embrapa Rondônia. Cultivo do abacaxi em Rondônia.

Nascimento, C. S. M. D. (2006). Resíduos gerados nas agroindústrias da microrregião de Propriá e as práticas de gestão ambiental adotadas.

Öktem, Y.A., Soylu, S.G.P. \& Aytan, N. (2012). The adsorption of methylene blue from aqueous solution by using waste potato peels; equilibrium and kinetic studies. Journal of Scientific and Industrial Research, 71, 817-821

Pathak, P. D., Mandavgane, S. A., \& Kulkarni, B. D. (2015). Fruit peel waste as a novel low-cost bio adsorbent. Reviews in Chemical Engineering, 31(4), 361-381.

Patil, S. D., Renukdas, S., \& Patel, N. T. (2012). Comparative study of kinetics of adsorption of methylene blue from aqueous solutions using cinnamon plant (Cinnamonum zeylanicum) leaf powder and pineapple (Ananas comosus) peel powder. Orbital-The Electronic Journal of Chemistry, 4(2), 77-100. 
Pavan, F.A., Mazzocato, A.C. \& Gushikem, Y. (2008). Removal of methylene blue dye from aqueous solutions by adsorption using yellow passion fruit peel as adsorbent. Bioresource technology, 99(8), pp.3162-3165.

Ponnusami, V., Vikram, S., \& Srivastava, S. N. (2008). Guava (Psidium guajava) leaf powder: novel adsorbent for removal of methylene blue from aqueous solutions. Journal of hazardous materials, 152(1), 276-286.

RANI, D.; NAND, K. (2004). Ensilage of pineapple processing waste for methane generation. Waste management, 24(5), p.523-528.

Reddy, P. M. K., Verma, P., \& Subrahmanyam, C. (2016). Bio-waste derived adsorbent material for methylene blue adsorption. Journal of the Taiwan Institute of Chemical Engineers, 58, 500508.

Ruangviriyachai, C., Niwaswong, C., Kosaikanon, N., Chanthai, S., \& Chaimart, P. (2010). Pineapple Peel Waste for Bioethanol Production. Journal of Biotechnology, (150), 10.

Sah, B. N. P.; Vasiljevic, V.; Mckechnie, S.; Donkor, O. N. (2015). Effect of pineapple waste powder on probiotic growth, antioxidant and antimutagenic activities of yogurt. Journal of Food Science and Technology, p.1-11.

Seader, J. D., Henley, E. J., Roper, D. K. (2010). Separation process principles (3 ed). John Wiley \& Sons.

SEBRAE. (2015). Agronegócio: fruticultura. Boletim de inteligência, 2015. Disponível em: < http://www.bibliotecas.sebrae.com.br> Acesso em 3 de maio de 2016.

SEBRAE. (2016). O cultivo e mercado do abacaxi. Disponível em: < http://www.sebrae.com.br> Acesso em 3 de maio de 2016.

Selvarajoo, A.; Hanson, S. (2014). Pyrolysis of pineapple peel: effect of temperature, heating rate and residence time on the biochar yield. In: Second Intl. Conf. on Advances in Applied Science and Environmental Engineering.

Senthil Kumar, P., Fernando, P. S. A., Ahmed, R. T., Srinath, R., Priyadharshini, M., Vignesh, A. M., \& Thanjiappan, A. (2014). Effect of Temperature on The Adsorption of Methylene Blue Dye onto Sulfuric Acid-Treated Orange Peel. Chemical Engineering Communications, 201(11), 1526-1547.

Subramaniam, R., \& Ponnusamy, S. K. (2015). Novel adsorbent from agricultural waste (cashew NUT shell) for methylene blue dye removal: optimization by response surface methodology. Water Resources and Industry, 11, 64-70.

Tayeb, A.M. and Hussein, D.S. (2015). Synthesis of TiO2 nanoparticles and their photocatalytic activity for Methylene Blue. American Journal of Nanomaterials, 3(2), pp.57-63.

Uddin, M. T., Islam, M. A., Mahmud, S., \& Rukanuzzaman, M. (2009). Adsorptive removal of methylene blue by tea waste. Journal of Hazardous Materials, 164(1), 53-60.

Viganó, J. (2012). Propriedades termodinâmicas de adsorção de água e cinética de secagem de subprodutos da industrialização de abacaxi (Ananás comosus L.): casca e cilindro central.

Wu, M.; Shiau, S. (2015). Effect of the Amount and Particle Size of Pineapple Peel Fiber on Dough Rheology and Steamed Bread Quality. Journal of Food Processing and Preservation, 39, p.549558. 